\title{
ELECTRON DIFFRACTION STUDY OF OLEOPHOBIC FILMS ON COPPER, IRON AND ALUMINUM*
}

\author{
L. O. Brockway \\ University of Michigan, Ann Arbor \\ and \\ J. Karle \\ T. S. Naval Research Laboratory, Washington, D. C. \\ Received February 9,1947
}

\section{INTRODUCTION}

The discovery and subsequent study by Zisman et al. (1) of oleophobic films adsorbed from solutions indicated that a variety of long hydrocarbon chains having polar end groups can be adsorbed from solutions in hydrocarbon solvents to give oriented films which are not wet by the solution. These films were readily made on a variety of solids. The films on platinum were demonstrated by Brockway and Livingston (2) to consist of layers of molecules oriented with their long axes approximately normal to the surface (usually less than $25^{\circ}$ from the normal) by using the electron diffraction technique.

The extension of diffraction studies of films to those on metals which are more reactive than platinum introduced several new factors. In the preparation of the surfaces the highly corrosive cleaning agents suitable for platinum could not be used, but new cleaning methods had to be developed. The surfaces after preparation were not the pure metal but a coating produced by the action of the cleaning agent or an oxide due to reaction with the atmosphere. The present study is concerned with three problems: (a) the preparation of smooth surfaces on copper, iron and aluminum and an electron diffraction examination of the surfaces for impurities, (b) the formation of films of stearic acid and $n$-octadecylamine on these surfaces and a diffraction examination of their structures, and (c) the comparison of these films with similar films on platinum. Previous electron diffraction examinations made on organic films on solids have not used adsorption from solution in the preparation of the films.

* The investigation reported here was part of a study of films on metals carried out at the University of Michigan for the Naval Research Laboratory under Contracts N173s-9051 and N173s-10452. 
Murison (3) studied films prepared by melting or spreading organic compounds on the surface and wiping off the excess. Germer and Storks (4) prepared uni- and multimolecular films of barium stearate and stearic acid by the Langmuir-Blodgett technique. Neither of these methods makes use of the property which is of special interest in the present investigation, i.e., the formation of oleophobic films from solutions of the film-forming material. The technique of dipping the metal into the solution, as was used in the work on platinum (1), had to be modified here as described below.

\section{The Preparation of Smooth Metal Surfaces}

The advantage of using smooth, flat metal surfaces in adsorbed film experiments is readily appreciated on consideration of the problem of determining the orientation of the hydrocarbon molecules composing such a film. Electron diffraction photographs provide data on the orientation of the molecules relative to a set of axes fixed in the specimen. With a flat surface the orientation between the surface and the molecule at the point of attachment is immediately deduced. It is apparent, however, that on a wavy or slightly rough surface it would not be possible to distinguish in an electron diffraction study between those variations in orientation which are characteristic of the attachment of the molecules and those variations due to non-uniformity of the surface. In fact, on a sufficiently rough surface an adsorbed film cannot maintain a regular orientation even over a small area because of the interference from abrupt changes in surface contour.

Another advantage of flatness is that it facilitates the determination of the chemical composition of the metal surface. From a surface uniformly covered by a thin layer of oxide, the electron diffraction pattern obtained will be characteristic of the oxide. If the surface is uniformly coated but rough, however, the pattern obtained may be characteristic of the metal as well as the oxide and under some circumstances the oxide pattern may be almost entirely obscured. This is due to the fact that a considerable portion of the electron scattering arises from transmission through the oxide-coated metal peaks which occur on a rough surface.

Metallographic polishing techniques were employed for the purpose of obtaining flat surfaces. The physical appearance of these surfaces was observed in a metallographic microscope and their chemical composition determined from electron diffraction reflection photographs. The identification of the diffraction patterns was accomplished by comparison with patterns obtained from known reference materials and with $\mathrm{X}$-ray data published by the American Society for Testing Materials. 


\section{Procedure}

The metals studied in the polishing experiments were copper ${ }_{2}$ iron and aluminum. The copper and aluminum were at least $99.90 \%$ pure. The iron was cold rolled steel-SAE 1020 (99.0\% iron). Metal samples about a centimeter square were polished on a metallographic wheel with selvyt cloths and shamva, levigated alumina or rouge. The same metal samples were used throughout the experiments.

Three different procedures were followed subsequent to the treatment with polishing agents. The metal samples were (a) rinsed with distilled water and dried in a stream of air, (b) rinsed with distilled water, wiped on a damp, grease-free paper towel, rinsed again with distilled water and dried in a stream of air, or (c) rinsed in distilled water, finished on a clean, damp selvyt polishing cloth running at about 200 r.p.m. for about 10 seconds and dried in a stream of air.

The samples examined by the electron diffraction technique were photographed within 15 minutes of their preparation with electrons accelerated by about 30 kilovolts.

\section{Results}

The study of the various polishing techniques has shown that the polishing agents adhere strongly to the metal surfaces and that vigorous action is required to remove the last traces. If a metal sample is only rinsed with distilled water following the polishing treatment, adhering particles of the polish are often seen with the naked eye and invariably under the microscope. This is true for the three metals studied, copper, iron and aluminum, and the three polishing agents, shamva, alumina and rouge. A gentle wipe with a damp paper towel removes most of the adhering polish but here and there small isolated clumps could be located under the microscope. The ten second finish on a clean selvyt cloth was sufficient to remove all visible traces of the polishing materials. These observations were substantiated by the electron diffraction studies of the surfaces as can be seen from the results included in Table I. The patterns obtained after a 10 second treatment on the auxiliary cloth showed the absence of polishing material. In the case of iron and copper, only a few vague indications of a crystalline pattern appear. After a somewhat longer treatment on the auxiliary cloth, 1-2 minutes, good crystalline patterns appear which are due to characteristic oxides of copper and iron. Since the vague arcs in the diffraction patterns appearing after the shorter standard treatment on copper and iron correspond to the darkest lines in the patterns after the longer treatment, it was concluded that the chemical nature of the surface after the standard treatment corresponds to that after the longer treatment and could thus be determined. For the 
short or long treatment on aluminum only an amorphous pattern is obtained. It is known that the natural oxide on aluminum which forms in air at room temperature is amorphous. The ability of the surfaces to form oleophobic films is independent of whether a short or long treatment time is used on the auxiliary cloth.

Typical diffraction results from the shamva polishing technique on copper and also some comparison data are shown in Fig. 1 as examples of

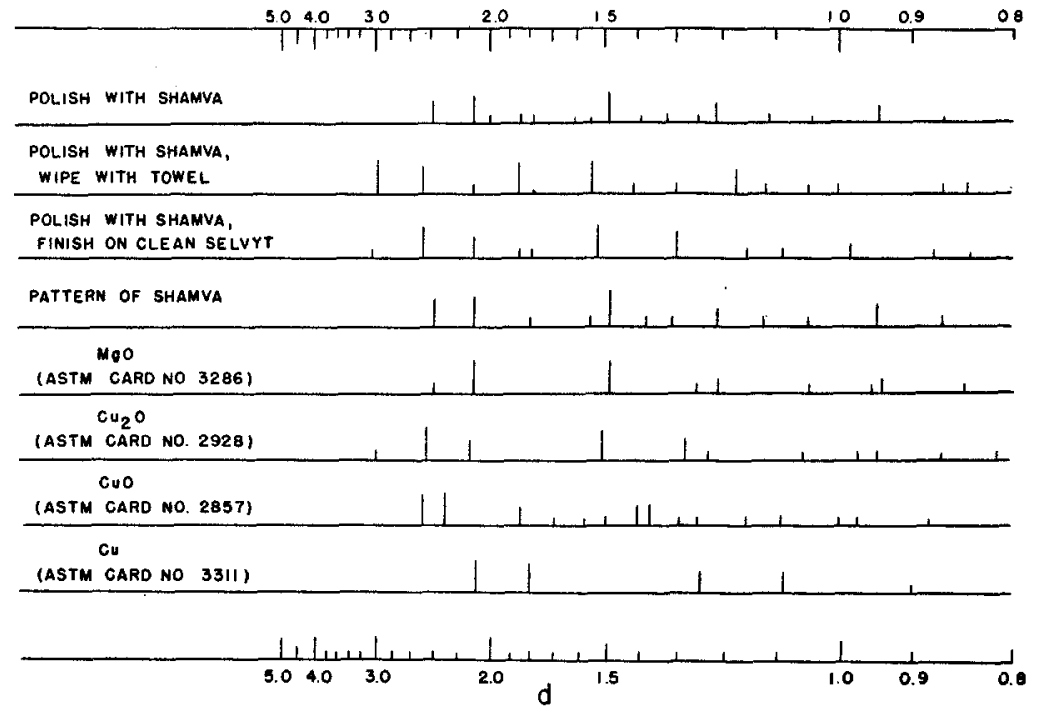

FIG. 1

Diffraction Patterns from Copper Surfaces Polished with Shamva and Patterns from Standard Comparison Materials

the information from which the results of Table I were compiled. The first pattern corresponds mainly to that of shamva, the polishing agent. The second and third patterns correspond mainly to a mixture of cuprous and cupric oxides. In no case was a pure metal surface obtained.

\section{The Formation of Oleophobic Films}

For the investigation of oleophobic film formation, metal surfaces were used which were prepared as described in the previous section. These surfaces were treated with solutions of stearic acid and $n$-octadecylamine in cetane forming adsorbed films which consisted of the polar additive. The orientation of the hydrocarbon chains in these adsorbed films is of considerable interest because of its relation to the physical properties of the films and to the nature of the binding to solid surfaces. Electron diffraction studies are able to give the orientation and packing of the mole- 
TABLE I

The Nature of the Electron Diffraction Patterns from the Polished Metal Surfaces

\begin{tabular}{|c|c|c|c|}
\hline Polishing technique & Copper & Iron & Aluminum \\
\hline Shamva I & shamva & shamva & shamva \\
\hline Shamva II & $\begin{array}{l}\mathrm{CuO} \text { and } \mathrm{Cu}_{2} \mathrm{O} \\
\text { or shamva }\end{array}$ & $\begin{array}{l}\text { iron oxide } \\
\text { or shamva }\end{array}$ & shamva \\
\hline Shamva III & $\mathrm{CuO}$ and $\mathrm{Cu}_{2} \mathrm{O}^{*}$ & $\gamma-\mathrm{FeOOH}{ }^{*}$ & $\begin{array}{l}\text { amorphous, } \\
\text { no pattern }\end{array}$ \\
\hline Rouge I & rouge & rouge & rouge \\
\hline Rouge II & rouge & rouge & rouge \\
\hline Rouge III & $\mathrm{CuO}$ and $\mathrm{Cu}_{2} \mathrm{O}^{*}$ & $\gamma-\mathrm{FeOOH}{ }^{*}$ & $\begin{array}{l}\text { amorphous, } \\
\text { no pattern }\end{array}$ \\
\hline Alumina I & alumina & alumina & alumina \\
\hline Alumina II & $\begin{array}{l}\mathrm{CuO} \text { and } \mathrm{Cu}_{2} \mathrm{O} \\
\text { or alumina }\end{array}$ & $\begin{array}{l}\text { iron oxide } \\
\text { or alumina }\end{array}$ & alumina \\
\hline Alumina III & $\mathrm{CuO}$ and $\mathrm{Cu}_{2} \mathrm{O}^{*}$ & $\gamma-\mathrm{FeOOH}^{*}$ & $\begin{array}{l}\text { amorphous, } \\
\text { no pattern }\end{array}$ \\
\hline
\end{tabular}

I Metallographic polishing followed by distilled water rinse.

II Metallographic polishing followed by wipe with towel and distilled water rinse.

III Metallographic polishing followed by further treatment for about ten seconds on clean polishing wheel and distilled water rinse.

* See text.

cules in adsorbed films. The theory of electron seattering from hydrocarbon films and its application to the determination of their structure has already been worked out $(3,4,5)$.

\section{Procedure}

Solutions were prepared of $n$-octadecylamine in cetane, $0.05 \%, 0.01 \%$ and $0.001 \%$ by weight and of stearic acid in cetane, $0.1 \%, 0.05 \%, 0.01 \%$ and $0.001 \%$ by weight. A drop of the $0.001 \%$ solution contains about enough polar molecules to cover the surface of the metal samples. The octadecylamine used was obtained from Armour and Company and further purified by recrystallizing the hydrochloride 4 times from ether. The amine was then obtained from its hydrochloride. The stearic acid used was obtained from the Eastman Kodak Company and the cetane was obtained from the du Pont Company. The stearic acid was not further purified but the cetane was recrystallized several times and percolated through alumina and silica to remove polar impurities. However, it contained a mixture of hydrocarbons with a range of chain lengths, m.p. $17.7-17.8^{\circ} \mathrm{C}$. The solutions were stored in pyrex glass-stoppered bottles 
which had been cleaned in a chromic-sulfuric acid bath, rinsed several times with hot distilled water and dried in a drying oven.

A drop of solution sufficient to cover the surface was placed on the specimens of copper, iron and aluminum by the following technique. A platinum scoop made of thin sheet was flamed, allowed to cool 15 seconds and then dipped into the desired solution. The drop picked up was then transferred to the metal surface. After an oleophobic film formed, the drop would draw away from the surface and occasionally roll off the edge to a considerable extent. With the more dilute solutions, the tendency to draw away from the surface was much less than that for concentrated solutions and would often need to be encouraged by tilting the metal sample. Any remaining liquid could readily be removed by touching the edge of a grease free paper towel to the edge of the drop. The technique of placing a drop of solution on the metals rather than dipping them into the liquid was chosen because of the danger of contaminating the solution with foreign material from the unpolished metal surfaces. No attempt was made to control the temperature of the experiments. It varied from $20-23^{\circ} \mathrm{C}$.

Some additional experiments were performed on thin rolled platinum, thin platinum soldered to a piece of steel so that it could be metallographically polished, and on metal surfaces abraded with $4 / 0$ emery paper. The possibility of forming oleophobic films from molten stearic acid was investigated and also some films were made with cerotic acid to study orientation effects with longer chains.

\section{Results}

The only polishing technique which gave good uniform results in oleophobic film formation was polishing technique (c) which involved a final treatment with a clean auxiliary polishing cloth. Copper and iron formed oleophobic films with all concentrations of the amine and acid solutions except $0.001 \%$. This result holds for each of the polishing agents, rouge, alumina and shamva. After a shamva polish using technique (c), aluminum forms films with all the solutions except the $0.001 \%$. No oleophobic films could be formed after using alumina or rouge on the aluminum, owing, presumably, to a roughening of the surface. If the polished metal was rinsed with distilled water or wiped with a paper towel after polishing, oleophobic films were not readily made and showed a lack of uniformity. The drop of solution would draw up slowly from the surface and leave it wet in spots. The polished metal surfaces used in film-forming experiments were wet by both water and cetane. The adsorbed films which were oleophobic to their parent solutions were also oleophobic to pure cetane and were not wet by water. 
It is interesting to note that a drop of pure cetane or of a very dilute solution of a polar additive, e.g., $0.001 \%$, can seriously interfere with the formation of an oleophobic film on a metal surface. If, after the drop is added, the excess liquid is drained from the surface and then a drop of concentrated solution which ordinarily gives a film in less than 20 seconds is added, it may taken an hour before an oleophobic film is formed. Apparently considerable time is required for the polar molecules to replace the cetane.

The time required to form oleophobic films on the copper, iron and aluminum is shortest for the more concentrated solutions. No great difference in speed was noted between the amine and acid. With the $0.1 \%$ and $0.05 \%$ solutions, the drop drew up on the metal surfaces within 30

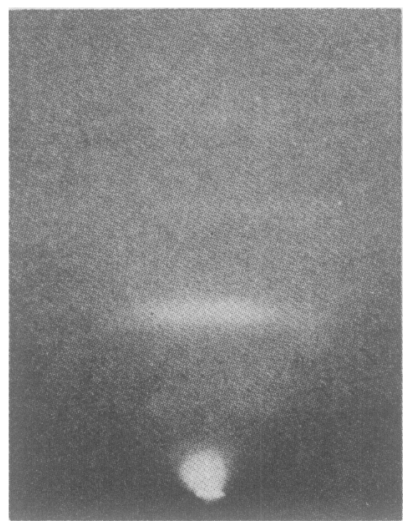

FIG. 2

Film from $0.01 \%$ Stearic Acid in Cetane on Metallographically Polished Iron

seconds at about $20^{\circ} \mathrm{C}$, whereas it took the $0.01 \%$ solutions from 2 to 10 minutes to do the same. The $0.001 \%$ solutions showed no tendency to form films after 24 hours in contact with a metal surface. There is a higher concentration limit in these experiments than in those of Bigelow, Pickett and Zisman (1) since the drop technique rather than the dipping technique was used here and the total available polar material is therefore less.

Whenever oleophobic films were formed from $n$-octadecylamine and stearic acid, the electron diffraction photographs showed layer lines nearly parallel to the shadow line. The odd orders showed intensity minima at their centers and the even orders showed maxima at their centers. No spots of high intensity appear on the layer lines (Fig. 2). From measuring the spacing between the layer lines it was found that the tilt of the axes of the molecules varies between $0^{\circ}$ and $10^{\circ}$ from a normal to the surface of attachment. The distribution of intensity within the orders of reflection 
shows that the molecules are oriented randomly about the surface normal and that they have a sufficiently random distribution on the surface so that there is no detectable intermolecular interference effect. Their distribution about their molecular axes cannot be discerned.

To investigate the effect of considerable roughness on oleophobic film formation, iron, copper and aluminum surfaces were abraded with 4/0 emery paper and attempts were made to form films. It was found that oleophobic films could be formed with $0.05 \% n$-octadecylamine and stearic acid solutions within a half hour to an hour at about $20^{\circ} \mathrm{C}$. only on copper and iron. Electron diffraction photographs give the surprising result that the molecules have a tilt which varies between $0^{\circ}$ and $10^{\circ}$ from a normal to the surface.

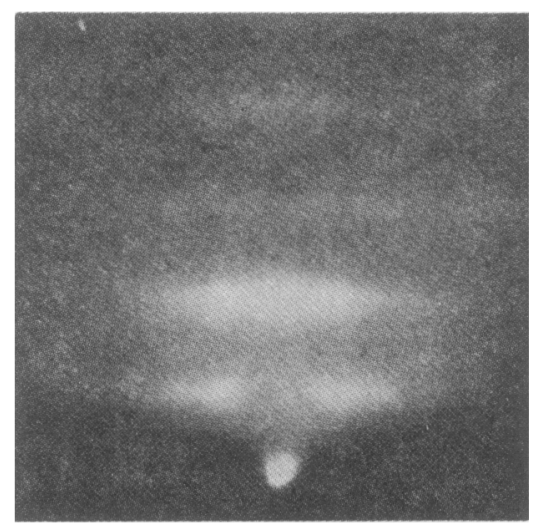

Fig. 3

Film from $0.05 \%$ Cerotic Acid in Cetane on Metallographically Polished Platinum

The possibility of forming oleophobic films on copper, iron and aluminum from molten stearic acid was investigated. The metals were polished with shamva and finished on an auxiliary cloth (technique (c)). If these metals are placed into molten stearic acid at $75^{\circ} \mathrm{C}$. a film is formed which is oleophobic to the molten acid and the metal may be removed dry. The film is also not wet by cetane or water. Electron diffraction photographs indicate that the molecules are tilted no more than $10^{\circ}$ from a vertical position.

In previous experiments on thin platinum having a bright and apparently smooth surface produced by rolling, oleophobic films were formed by dipping the metal into the desired solution. This technique was suitable since the platinum could be readily cleaned in a chromicsulfuric acid bath and subsequently rinsed and flamed. A comparison of the dipping technique with the drop technique used on copper, iron and 
aluminum was considered desirable. In addition, an investigation was made to determine the role played by the previous preparation of the platinum surface by comparing some results on thin flamed platinum with those on metallographically polished platinum. With both the drop and dipping techniques on thin flamed platinum an oleophobic film is formed with $0.05 \%$-octadecylamine in cetane or $0.1 \%$ stearic acid in cetane in a few seconds. The electron diffraction results show that the axes of the molecules vary from $0^{\circ}$ to $30^{\circ}$ from a normal to the surface as a whole. However, the drop technique on metallographically polished platinum gave films in a few seconds in which the axes of the molecules were oriented within $10^{\circ}$ of the surface normal. This is similar to the results on metallographically polished copper, iron and aluminum. It was

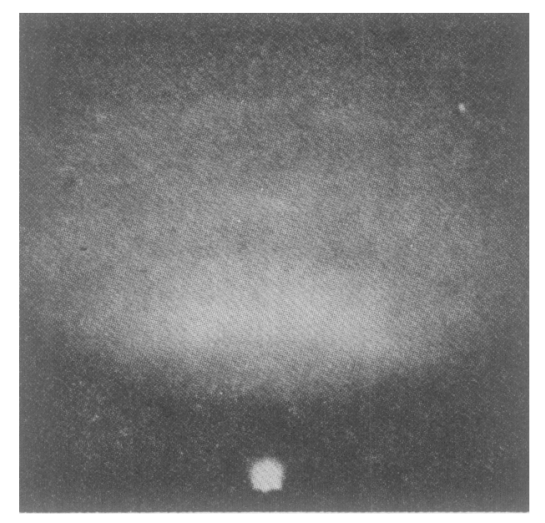

FIG. 4

Film from $0.05 \%$ Cerotic Acid in Cetane on Thin Rolled Platinum

also found that $0.05 \%$ cerotic acid solution in cetane produces an oleophobic film on metallographically polished platinum in which the molecular axes are parallel within a degree or two to the surface normal whereas on thin rolled platinum it forms a film in which the molecular axes vary from $0^{\circ}$ to $30^{\circ}$ from the surface normal (5b) (Figs. 3 and 4 ). A practically vertical orientation was also found for the molecules in a film of cerotic acid on metallographically polished iron.

\section{Discussion and Conclusions}

An important surface feature for facilitating the formation of oleophobic films is smoothness. The role played by smoothness can be understood from a consideration of the structure of oleophobic films. In the experiments performed on copper, iron and aluminum and metallographically polished platinum the molecules in the films formed from $n$ - 
octadecylamine and also stearic acid showed an average tilt of $5^{\circ}$ from the surface normal. The electron diffraction results comparing the structure of films on thin rolled platinum to that on metallographically polished platinum indicate that the larger deviations from a vertical position on thin platinum is not due to an inherent structural characteristic of the film but is rather a measure of the submicroscopic unevenness of the platinum surface. If film formation is carried on for several hours on the thin platinum, a film is formed whose molecules are parallel to the normal to the surface as a whole (2). In addition it should be noted that it is not impossible to obtain oleophobic films on roughened surfaces as was shown with surfaces abraded with $4 / 0$ emery paper. However, the time of formation is about 0.5 hour for a $0.1 \%$ solution of stearic acid in cetane instead of a few seconds and the molecules in the resulting film show a variation in orientation no greater than $10^{\circ}$ from the normal to the surface as a whole. It is quite apparent from the experiments on the thin platinum and the abraded surfaces that the final equilibrium orientations of the molecules in an oleophobic film are not completely governed by the underlying surface contour. The significance of an orientation normal to the surface as a whole is that the molecules of such a film are perpendicular to the film-oil interface. Since this structural requirement is readily satisfied on a smooth surface, the importance of smoothness is now clear. It may be noted that, from observations on oleophobic behavior, Bigelow, Pickett and Zisman (1) suggested that such films consist of fairly closely packed molecules standing normal to the surface.

It has been found, in agreement with previous results, that with smoother surfaces, lower temperatures and more concentrated solutions the rate of oleophobic film formation is increased. A precise study of rate was not made, however, as some of the variables upon which the rate depends were not carefully controlled. The rate is not critically dependent upon the chemical nature of the solid surfaces investigated nor the nature of the end group on the polar molecules considered. The smooth surfaces of copper, iron and aluminum consisted of oxides of the metals, but neither the rate of film formation nor the structure of the film on these surfaces differs appreciably from the films on really smooth platinum. The similarity of the results from copper, iron, aluminum and platinum surfaces indicates a lack of any specific chemical effect in the adsorption.

Before a complete understanding of oleophobic behavior may be obtained several additional questions concerning the structural features of the adsorbed films need to be answered. These concern whether the film is a uniform monolayer free from solvent molecules and, if so, whether all its constituent molecules occur with their polar groups oriented toward the surface of the solid. The hydrocarbon end of the polar molecule may have a considerable attraction for the surface, as is indicated by the 
poisoning effect of cetane and dilute solutions made with cetane mentioned above. So far it has not been possible to decide in general upon the number of layers, the position of the polar groups, nor the amount of solvent in oleophobic films. An experiment performed with a solution of $n$-octadecylamine in dicyclohexyl has indicated that oleophobic films formed from this solution consist of mono-layers (1).

\section{ACKNOWLEDGMENTS}

This investigation has benefited largely from frequent conferences with Dr. W. A. Zisman whose continued interest has been most stimulating. Mr. W. C. Bigelow purified the cetane and $n$-octadecylamine used in these experiments. The interest and support provided by the Naval Research Laboratory in the study of films on surfaces is gratefully acknowledged.

\section{REFERENCES}

1. Bigelow, W. C., Pickett, D. L., and Zisman, W. A., J. Colloid Sci. 1, 513 (1946); Part II, to be published.

2. Brockway, L. O., and Livingston, R. L., to be published.

3. Murison, C. A., Phil. Mag. 17, 201 (1934).

4. Germer, L. H., and Storks, K. H., J. Chem. Phys. 6, 280 (1938).

5. (a) Karle, J., J. Chem. Phys. 14, 297 (1946). (b) Karle, J., and Brockway, L. O., to be published. 